\title{
The Generalized Taxicab Group
}

\author{
Harun Barış Çolakoğlu*
}

(Communicated by Yusuf Yaylı)

\begin{abstract}
In this study, we determine the generalized taxicab group consisting all isometries of the real plane endowed with the generalized taxicab metric. First we develop natural analogues of Euclidean reflection and rotation notions, and then determine all isometries in the generalized taxicab plane. Finally, we show that the generalized taxicab group is semidirect product of the translation group and the generalized taxicab symmetry group of the unit generalized taxicab circle, as Euclidean group. We also see that there are transformations of the real plane onto itself which preserve the generalized taxicab distance, but not preserve the Euclidean distance.
\end{abstract}

Keywords: Taxicab metric; generalized taxicab metric; distance; angle measure; reflection; rotation; isometry; the generalized taxicab group.

AMS Subject Classification (2010): 51K05, 51K99, 51N99.

\section{Introduction}

The taxicab metric was given in a family of metrics of the real plane by Minkowski. Using this metric, taxicab geometry was introduced by Menger [10], and developed by Krause [8]. In [15], Lawrance J. Wallen altered the taxicab metric by redefining in order to get rid of imperative symmetry, and called it the (slightly) generalized taxicab metric. During the recent years, metric geometries based on these metrics have been studied and developed in many directions. See [1], [2], [3], [4], [5], [6], [7], [12], [13], and [14] for some of studies.

Let $P_{1}=\left(x_{1}, y_{1}\right)$ and $P_{2}=\left(x_{2}, y_{2}\right)$ be two points in $\mathbb{R}^{2}$. For each positive real numbers $a$ and $b$, the function $d_{T_{g}}: \mathbb{R}^{2} \times \mathbb{R}^{2} \rightarrow[0, \infty)$ defined by

$$
d_{T_{g}}\left(P_{1}, P_{2}\right)=a\left|x_{1}-x_{2}\right|+b\left|y_{1}-y_{2}\right|
$$

is called generalized taxicab distance function in $\mathbb{R}^{2}$, and the real number $d_{T_{g}}\left(P_{1}, P_{2}\right)$ is called generalized taxicab distance between points $P_{1}$ and $P_{2}$, while the well-known Euclidean distance between points $P_{1}$ and $P_{2}$ is

$$
d_{E}\left(P_{1}, P_{2}\right)=\left(\left(x_{1}-x_{2}\right)^{2}+\left(y_{1}-y_{2}\right)^{2}\right)^{1 / 2} .
$$

The real plane endowed with the generalized taxicab metric is called the generalized taxicab plane, and this metric space is denoted by $\mathbb{R}_{T_{q}}^{2}$. An isometry is a transformation of a metric space onto itself which preserves distances, and one of the basic geometric problems for a given metric space is to determine the group of isometries of it. It is well-known that the group of all isometries of the Euclidean plane is the Euclidean group $E(2)$ which is semidirect product of the translation group $T(2)$ consisting of all translations, and the symmetry group of unit circle $O(2)$. In [3], Euclidean isometries that preserve the generalized taxicab distance are studied. In this study, our main aim is to determine the all isometries of $\mathbb{R}_{T_{g}}^{2}$ and the generalized taxicab isometry group $T_{g}(2)$ related to them. To do that, we first define angle measurement in the generalized taxicab plane, then we develop natural analogues of Euclidean reflection and rotation notions in the generalized taxicab plane. Finally, we see that $T_{g}(2)$ is semidirect product of the translation group $T(2)$ consisting of all translations, and the generalized taxicab symmetry group of the unit generalized taxicab circle $O_{T_{g}}(2)$, as Euclidean group. Here, we also see that there are transformations of the real plane onto itself which preserve the generalized taxicab distance, but

Received : 28-04-2018, Accepted : 17-09-2018

* Corresponding author 
not preserve Euclidean distance. Throughout the paper, we sometimes use $g t$ - prefix for generalized taxicab to shorten phrases.

The following proposition states an equation which relates the Euclidean distance to the generalized taxicab distance between two points in the Cartesian coordinate plane (see also [3]):

Proposition 1.1. For any two points $P_{1}$ and $P_{2}$ in $\mathbb{R}^{2}$ that do not lie on a vertical line, if $m$ is the slope of the line through $P_{1}$ and $P_{2}$, then

$$
d_{T_{g}}\left(P_{1}, P_{2}\right)=\mu(m) d_{E}\left(P_{1}, P_{2}\right)
$$

where $\mu(m)=(a+b|m|) /\left(1+m^{2}\right)^{1 / 2}$. If $P_{1}$ and $P_{2}$ lie on a vertical line, then

$$
d_{T_{g}}\left(P_{1}, P_{2}\right)=b d_{E}\left(P_{1}, P_{2}\right) .
$$

Proof. Let $P_{1}=\left(x_{1}, y_{1}\right)$ and $P_{2}=\left(x_{2}, y_{2}\right)$ such that $x_{1} \neq x_{2}$. Then $m=\left(y_{2}-y_{1}\right) /\left(x_{2}-x_{1}\right)$. Clearly, $d_{T_{g}}\left(P_{1}, P_{2}\right)=$ $\left|x_{1}-x_{2}\right|(a+b|m|)$ and $d_{E}\left(P_{1}, P_{2}\right)=\left|x_{1}-x_{2}\right|\left(1+m^{2}\right)^{1 / 2}$, thus we have $d_{T_{g}}\left(P_{1}, P_{2}\right)=\mu(m) d_{E}\left(P_{1}, P_{2}\right)$, where $\mu(m)=(a+b|m|) /\left(1+m^{2}\right)^{1 / 2}$. If $x_{1}=x_{2}$, then $d_{T_{g}}\left(P_{1}, P_{2}\right)=b\left|y_{1}-y_{2}\right|$ and $d_{E}\left(P_{1}, P_{2}\right)=\left|y_{1}-y_{2}\right|$, thus we have Equation (1.4).

The following two corollaries follow directly from Proposition 1.1:

Corollary 1.1. Let $P_{1}, P_{2}, P_{3}$ and $P_{4}$ be four points in $\mathbb{R}^{2}$. If lines $P_{1} P_{2}$ and $P_{3} P_{4}$ are coincident, parallel or symmetric about a line parallel to an axis; in addition for $a=b$, if lines $P_{1} P_{2}$ and $P_{3} P_{4}$ are perpendicular, then

$$
d_{T_{g}}\left(P_{1}, P_{2}\right)=d_{T_{g}}\left(P_{3}, P_{4}\right) \text { if and only if } d_{E}\left(P_{1}, P_{2}\right)=d_{E}\left(P_{3}, P_{4}\right) .
$$

Corollary 1.2. In $\mathbb{R}^{2}$, if $P_{1}$ and $P_{2}$ two distinct points and $X$ is a point on the line $P_{1} P_{2}$, and distinct from $P_{1}$ and $P_{2}$, then

$$
d_{T_{g}}\left(P_{1}, X\right) / d_{T_{g}}\left(X, P_{2}\right)=d_{E}\left(P_{1}, X\right) / d_{E}\left(X, P_{2}\right) .
$$

\section{The Generalized Taxicab Angle Measurement}

To measure angles in the generalized taxicab plane, we will mirror the usual Euclidean radian notion, as in [13] (see also [1] and [7] for angle measurement functions in the taxicab plane). So, we define angle measurement on the unit generalized taxicab circle which is shown in Figure 1.

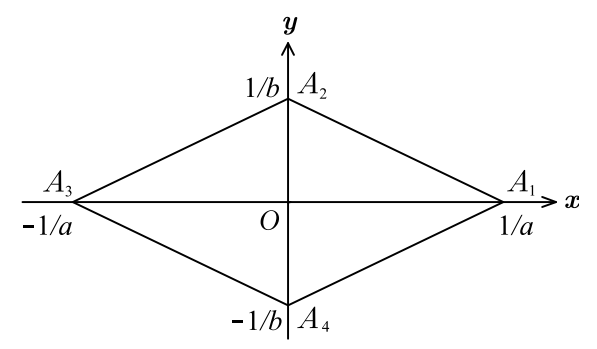

Figure 1: Unit generalized taxicab circle (for $a=1 / 5$ and $b=1 / 3$ ).

Definition 2.1. One generalized taxicab radian (gt-radian) is the measure of a positive directed central angle that intercepts a gt-arc of gt-length of 1 in the unit gt-circle. The measure $\theta$ in gt-radian of an angle is the number of gt-radians subtended by the angle on the unit gt-circle about the vertex.

As usual, we take angles with counterclockwise orientation as positive measure, and denote the measure in gt-radians of directed angle $P_{1} O P_{2}$ by $m_{T_{g}}\left(P_{1} O P_{2}\right)$ (see Figure 2).

It follows that the unit gt-circle has 8 gt-radians since it has circumference 8 . Since all distances along a gt-circle are scaled equally as the radius changed, for a central angle $\theta$ of any gt-circle, the gt-length $s$ of the gt-arc intercepted on a circle of radius $r$ by the angle, is given by $s=r \theta$, as in Euclidean geometry. Notice that the ratio of the circumference of any generalized taxicab circle to its diameter is $\pi_{T_{g}}=4$. 


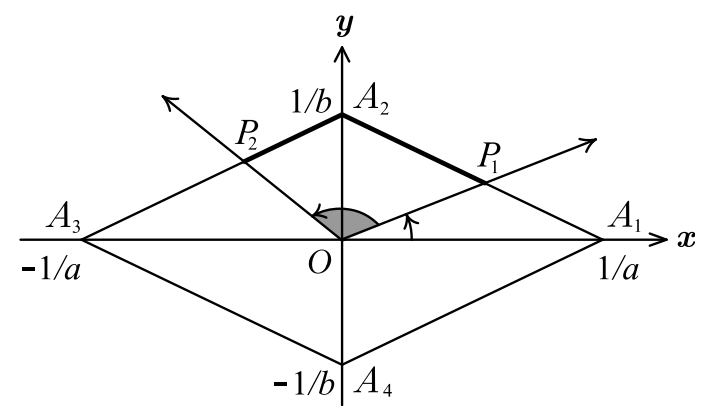

Figure 2: The generalized taxicab measures of positive directed angles:

$$
m_{T_{g}}\left(A_{1} O P_{1}\right)=d_{T_{g}}\left(A_{1}, P_{1}\right), m_{T_{g}}\left(P_{1} O P_{2}\right)=d_{T_{g}}\left(P_{1}, A_{2}\right)+d_{T_{g}}\left(A_{2}, P_{2}\right) .
$$

The following proposition states a formula to calculate measure in gt-radians of positive directed angles in standard position:

Proposition 2.1. Let $P=(x, y)$ be a point on the unit gt-circle. Then, measure in gt-radians of positive directed angle $A_{1} O P$ is

$$
m_{T_{g}}\left(A_{1} O P\right)= \begin{cases}2-2 a x & , \text { if } P \in\left[A_{1}, A_{2}\right] \cup\left[A_{2}, A_{3}\right] \\ 6+2 a x & \text {, if } P \in\left[A_{3}, A_{4}\right] \cup\left[A_{4}, A_{1}\right]\end{cases}
$$

Proof. Using definitions of the gt-distance and the gt-radian, one has the following equation:

$$
m_{T_{g}}\left(A_{1} O P\right)=\left\{\begin{array}{cl}
a\left|x-\frac{1}{a}\right|+b|y| & , \text { if } P \in\left[A_{1}, A_{2}\right] \\
2+a|x|+b\left|y-\frac{1}{b}\right| & , \text { if } P \in\left[A_{2}, A_{3}\right] \\
4+a\left|x+\frac{1}{a}\right|+b|y| & , \text { if } P \in\left[A_{3}, A_{4}\right] \\
6+a|x|+b\left|y+\frac{1}{b}\right| & , \text { if } P \in\left[A_{4}, A_{1}\right]
\end{array}\right.
$$

In addition, if $P \in\left[A_{1}, A_{2}\right]$, then $y=(1-a x) / b$; if $P \in\left[A_{2}, A_{3}\right]$, then $y=(1+a x) / b$; if $P \in\left[A_{3}, A_{4}\right]$, then $y=$ $(-1-a x) / b$; if $P \in\left[A_{4}, A_{1}\right]$, then $y=(a x-1) / b$. Using these values in equation above, one gets Equation (2.1).

Notice that having the same measure in gt-radians is not enough for angles to be Euclidean congruent since not every Euclidean congruent angles have the same measure in gt-radians. It is easy to see that opposite angles always have the same measure in gt-radians and straight angles always have measure of 4 gt-radians. Besides, if $a=b$, then Euclidean right angles always have measure of 2 gt-radians. But if $a \neq b$, the only Euclidean right angles with measure of 2 gt-radians are angles whose sides are parallel to coordinate axes (see Figure 3 ). Let us call an angle with measure of 2 gt-radians, gt-right angle. Thus, we naturally have gt-perpendicular notion, too: In Figure 3, the line $Q_{1} Q_{2}$ is the gt-perpendicular bisector of the line segment $\left[P_{1} P_{2}\right]$, since $m_{T_{g}}\left(P_{1} O Q_{1}\right)=2$ and $d_{T_{g}}\left(P_{1}, O\right)=d_{T_{g}}\left(O, P_{2}\right)$, and similarly the line $P_{1} P_{2}$ is the gt-perpendicular bisector of the line segment $\left[Q_{1} Q_{2}\right]$, since $m_{T_{g}}\left(P_{1} O Q_{1}\right)=2$ and $d_{T_{g}}\left(Q_{1}, O\right)=d_{T_{g}}\left(O, Q_{2}\right)$. It is not difficult to see that if lines $l_{1}$ and $l_{2}$ with slope $m_{1}$ and $m_{2}$ are gt-perpendicular, then $m_{1} m_{2}=-\frac{a^{2}}{b^{2}}$.

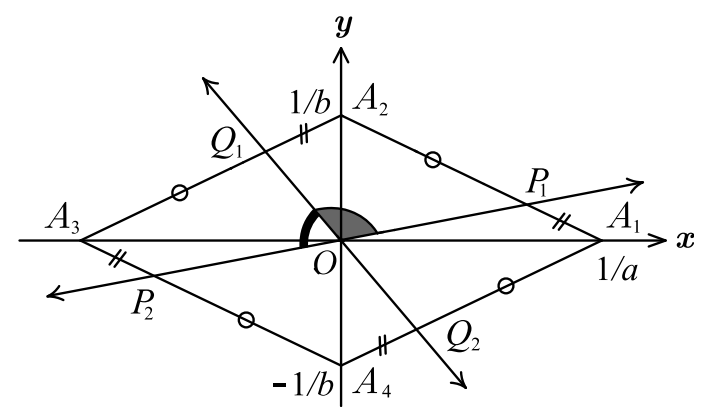

Figure 3: The generalized taxicab right angles: $m_{T_{g}}\left(P_{1} O Q_{1}\right)=m_{T_{g}}\left(Q_{1} O P_{2}\right)=m_{T_{g}}\left(P_{2} O Q_{2}\right)=m_{T_{g}}\left(Q_{2} O P_{1}\right)=\pi_{T_{g}} / 2=2$ gt-radians. 


\section{The Generalized Taxicab Reflections}

We take the natural analogue of Euclidean reflection for the generalized taxicab version:

Definition 3.1. The generalized taxicab reflection $\sigma_{\alpha}$ in line $\alpha$ is the mapping of $\mathbb{R}_{T_{g}}^{2}$ such that $\sigma_{\alpha}(X)=X$ if point $X$ is on $\alpha$, and $\sigma_{\alpha}(X)=Y$ if point $X$ is off $\alpha$ and $\alpha$ is the gt-perpendicular bisector of the line segment $[X Y]$. If $\sigma_{\alpha}(X)=Y$, then $Y$ is called gt-symmetry of the point $X$ about the line $\alpha$.

Since gt-perpendicular bisector for the line segment $X Y$ is different from the Euclidean analogue, we need to determine a way to find $\sigma_{\alpha}(X)$ for a given line $\alpha$ and any point $X$ : Given a line $\alpha$ and any point $X$. If $X$ is on $\alpha$, then $\sigma_{\alpha}(X)=X$ by definition. Let $X$ be off $\alpha$. First, draw the unit gt-circle with center $X$, then for a point $P$ such that the ray $\left[X P\right.$ is parallel to $\alpha$, determine the point $P^{\prime}$ such that $m_{T_{g}}\left(P X P^{\prime}\right)=2$ gt-radians (see Figure 4). Thus, the line $P^{\prime} X$ is gt-perpendicular to $\alpha$. If $I$ is the intersection point of the line $P^{\prime} X$ and $\alpha$, then the gt-symmetric point of $X$ with respect to the point $I$ (clearly, gt-symmetry about a point is the same as the Euclidean symmetry about a point) is $\sigma_{\alpha}(X)=Y$, since $d_{T_{g}}(X, I)=d_{T_{g}}(I, Y)$ by Corollary 1.2, and so $\alpha$ is the gt-perpendicular bisector of the line segment $[X Y]$. Note that gt-reflection in line parallel to $y=0$ or $x=0$, and gt-reflection in line parallel to $y=x$ or $y=-x$ for $a=b$, are the same as their Euclidean analogues. In addition, $\sigma_{\alpha}$ fixes every point on $\alpha ; \sigma_{\alpha}$ fixes line $\beta$ if and only if $\beta=\alpha$ or $\beta$ is gt-perpendicular to $\alpha$; if $\alpha_{1}$ and $\alpha_{1}$ are parallel, then $\sigma_{\alpha_{1}} \sigma_{\alpha_{2}}$ is a translation; and $\sigma_{\alpha} \sigma_{\alpha}=I$, as in Euclidean geometry.

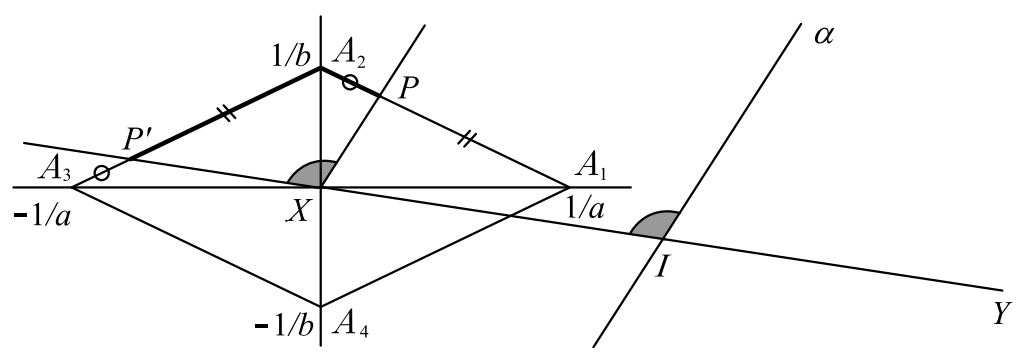

Figure 4: $Y$ is the image of the point $X$ under the gt-reflection $\sigma_{\alpha}$.

To determine isometric gt-reflections of $\mathbb{R}_{T_{g}}^{2}$, let us consider gt-reflections in lines through origin. Let $\sigma_{l}$ be a gt-reflection in a line $l$ through origin, and let $\mathcal{C}$ be a gt-circle with center $O$. Clearly, if $\sigma_{l}(X)$ is off $\mathcal{C}$ for a point $X$ is on $\mathcal{C}$, then $\sigma_{l}$ is not an isometry. Besides, if $\sigma_{l}$ is an isometric gt-reflection in line through origin, then $\sigma_{l}$ fixes $\mathcal{C}$. One can see that gt-reflection in line $y=0, x=0, y=\frac{a}{b} x$ or $y=-\frac{a}{b} x$ fixes gt-circles with center $O$. To be precise, let $P_{1}=\left(x_{1}, y_{1}\right)$ and $P_{2}=\left(x_{2}, y_{2}\right)$ two points, then $\sigma_{y=0}\left(P_{i}\right)=\left(x_{i},-y_{i}\right), \sigma_{x=0}\left(P_{i}\right)=\left(-x_{i}, y_{i}\right)$, $\sigma_{y=\frac{a}{b} x}\left(P_{i}\right)=\left(\frac{b}{a} y_{i}, \frac{a}{b} x_{i}\right)$, and $\sigma_{y=-\frac{a}{b} x}\left(P_{i}\right)=\left(-\frac{b}{a} y_{i},-\frac{a}{b} x_{i}\right)$ for $i=1,2$. Using the definition of the generalized taxicab distance between two points one gets $d_{T_{g}}\left(P_{1}, P_{2}\right)=d_{T_{g}}\left(\sigma_{l}\left(P_{1}\right), \sigma_{l}\left(P_{2}\right)\right)$ where $l$ is $y=0, x=0, y=\frac{a}{b} x$ or $y=-\frac{a}{b} x$. Thus, a gt-reflection in line parallel to $y=0, x=0, y=\frac{a}{b} x$ or $y=-\frac{a}{b} x$ is an isometry. One can also see easily that the other gt-reflections in a line through origin are not isometries, since they do not fix gt-circles with center $O$. Therefore, a gt-reflection in line not parallel to $y=0, x=0, y=\frac{a}{b} x$ or $y=-\frac{a}{b} x$ is not an isometry. Notice that if $a=b$ then isometric gt-reflections $\sigma_{y=0}, \sigma_{x=0}, \sigma_{y=\frac{a}{b} x}$ and $\sigma_{y=-\frac{a}{b} x}$ preserve also the Euclidean distances. If $a \neq b$ then isometric gt-reflections $\sigma_{y=0}$ and $\sigma_{x=0}$ preserve also the Euclidean distances, but isometric gt-reflections $\sigma_{y=\frac{a}{b} x}$ and $\sigma_{y=-\frac{a}{b} x}$ do not preserve the Euclidean distances, while they preserve the generalized taxicab distances.

Now, we state following proposition as a result of our observations above:

Proposition 3.1. In the generalized taxicab plane, the set of isometric gt-reflections in lines through origin is

$$
S_{T_{g}}=\left\{\sigma_{y=0}, \sigma_{x=0}, \sigma_{y=\frac{a}{b} x}, \sigma_{y=-\frac{a}{b} x}\right\} .
$$

One can also write the isometric gt-reflections in matrix form as:

$$
S_{T_{g}}=\left\{\left[\begin{array}{cc}
1 & 0 \\
0 & -1
\end{array}\right],\left[\begin{array}{cc}
-1 & 0 \\
0 & 1
\end{array}\right],\left[\begin{array}{cc}
0 & \frac{b}{a} \\
\frac{a}{b} & 0
\end{array}\right],\left[\begin{array}{cc}
0 & -\frac{b}{a} \\
-\frac{a}{b} & 0
\end{array}\right]\right\}
$$

www.iejgeo.com 


\section{The Generalized Taxicab Rotations}

Similarly, we take the natural analogue of Euclidean rotation for the generalized taxicab version:

Definition 4.1. The generalized taxicab rotation $\rho_{C, \theta}$ about point $C$ through directed angle of $\theta$ in gt-radians is the mapping of $\mathbb{R}_{T_{g}}^{2}$ such that $\rho_{C, \theta}(C)=C$, and $\rho_{C, \theta}(X)=Y$ for $X \neq C$, where $d_{T_{g}}(C, Y)=d_{T_{g}}(C, X)$ and $\theta=m_{T_{g}}(X C Y)$.

Since gt-distance function and measure of angle in gt-radians are different from Euclidean analogues, we need to determine a way to find $\rho_{C, \theta}(X)$ for given points $C, X$ and directed angle measure in gt-radian $\theta$ : Given points $C, X$ and directed angle measure in gt-radian $\theta$. If $X=C$, then $\rho_{C, \theta}(X)=C$, by the definition. Let $X \neq C$. First, we draw the unit gt-circle $\mathcal{C}_{1}$ with center $C$ and the gt-circle $\mathcal{C}_{2}$ with center $C$ and radius $[C X]$, then we take the intersection point $P$ of the ray $\left[C X\right.$ and the gt-circle $\mathcal{C}_{1}$. Thus, for the point $P^{\prime}$ such that $m_{T_{g}}\left(P C P^{\prime}\right)=\theta$, the intersection point of the ray $\left[C P^{\prime}\right.$ and the gt-circle $\mathcal{C}_{2}$ is $Y=\rho_{C, \theta}(X)$, since $\theta=m_{T_{g}}(X C Y)$ and $d_{T_{g}}(C, Y)=d_{T_{g}}(C, X)$ (see Figure 5). Note that gt-rotation about a point through 4 gt-radians, and gtrotation about a point through 2 gt-radians for $a=b$, are the same as their Euclidean analogues. In addition, a nonidentity gt-rotation fixes exactly one point (its center); a gt-rotation with center $C$ fixes every gt-circle with center $C$; and $\rho_{C, \theta_{2}} \rho_{C, \theta_{1}}=\rho_{C, \theta_{1}+\theta_{2}}$, as in Euclidean geometry.

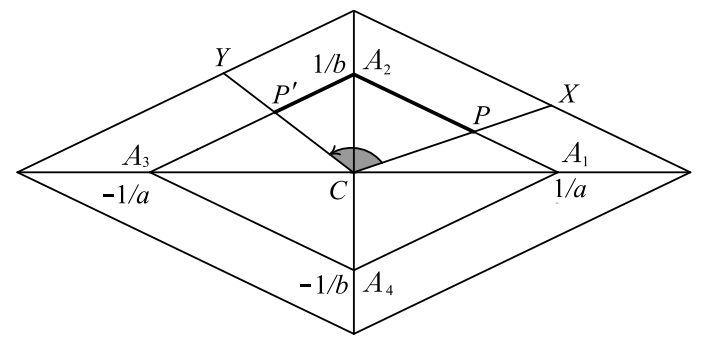

Figure 5: $Y$ is the image of the point $X$ under the gt-rotation $\rho_{C, \theta}$.

To determine isometric gt-rotations of $\mathbb{R}_{T_{g}}^{2}$, let us consider gt-rotations about origin through directed angle of $\theta \in[0,8)$, for the points $A_{1}$ and $A_{2}$ of the unit gt-circle. Clearly, if $\sigma_{O, \theta}$ is an isometric gt-rotation, then $d_{T_{g}}\left(\rho_{O, \theta}\left(A_{1}\right), \rho_{O, \theta}\left(A_{2}\right)\right)$ $=2$ since $d_{T_{g}}\left(A_{1}, A_{2}\right)=2$. One can see that $d_{T_{g}}\left(\rho_{O, \theta}\left(A_{1}\right), \rho_{O, \theta}\left(A_{2}\right)\right)=2$ for $\theta \in\{0,2,4,6\}$. To be precise, let $P_{1}=\left(x_{1}, y_{1}\right)$ and $P_{2}=\left(x_{2}, y_{2}\right)$ two points, then $\rho_{O, 0}\left(P_{i}\right)=\left(x_{i}, y_{i}\right), \rho_{O, 2}\left(P_{i}\right)=\left(-\frac{b}{a} y_{i}, \frac{a}{b} x_{i}\right)$, $\rho_{O, 4}\left(P_{i}\right)=\left(-x_{i},-y_{i}\right)$, and $\rho_{O, 6}\left(P_{i}\right)=\left(\frac{b}{a} y_{i},-\frac{a}{b} x_{i}\right)$ for $i=1,2$. Using the definition of the generalized taxicab distance between two points one gets $d_{T_{g}}\left(P_{1}, P_{2}\right)=d_{T_{g}}\left(\rho_{O, \theta}\left(P_{1}\right), \rho_{O, \theta}\left(P_{2}\right)\right)$ for $\theta \in\{0,2,4,6\}$. Thus, a gtrotation about a point $C$ through directed angle of $\theta \in\{0,2,4,6\}$ in gt-radians is an isometry. One can also see easily that the other gt-rotations about origin are not isometries, since $d_{T_{g}}\left(\rho_{O, \theta}\left(A_{1}\right), \rho_{O, \theta}\left(A_{2}\right)\right)$ $<2$ for $\theta \in[0,8)-\{0,2,4,6\}$, due to points $\rho_{O, \theta}\left(A_{1}\right)$ and $\rho_{O, \theta}\left(A_{2}\right)$ are not on opposite sides of the unit gt-circle. Therefore, a gt-rotation about a point $C$ through $\theta \in[0,8)-\{0,2,4,6\}$ is not an isometry.

Notice that if $a=b$ then isometric gt-rotations $\rho_{C, 0}, \rho_{C, 2}, \rho_{C, 4}$ and $\rho_{C, 6}$ preserve also the Euclidean distances. If $a \neq b$ then isometric gt-rotations $\rho_{C, 0}$ and $\rho_{C, 4}$ preserve also the Euclidean distances, but isometric gt-rotations $\rho_{C, 2}$ and $\rho_{C, 6}$ do not preserve the Euclidean distances, while they preserve the generalized taxicab distances.

Now, we state following proposition as a result of our observations above:

Proposition 4.1. In the generalized taxicab plane, the set of isometric gt-rotations about origin for $\theta \in[0,8)$ is

$$
R_{T_{g}}=\left\{\rho_{O, 0}, \rho_{O, 2}, \rho_{O, 4}, \rho_{O, 6}\right\} .
$$

One can also write the isometric gt-rotations in matrix form as:

$$
R_{T_{g}}=\left\{\left[\begin{array}{ll}
1 & 0 \\
0 & 1
\end{array}\right],\left[\begin{array}{cc}
0 & -\frac{b}{a} \\
\frac{a}{b} & 0
\end{array}\right],\left[\begin{array}{cc}
-1 & 0 \\
0 & -1
\end{array}\right],\left[\begin{array}{cc}
0 & \frac{b}{a} \\
-\frac{a}{b} & 0
\end{array}\right]\right\} .
$$

One can see that the composition of any two isometries in $S_{T_{g}} \cup R_{T_{g}}$ is also in $S_{T_{g}} \cup R_{T_{g}}$ : 


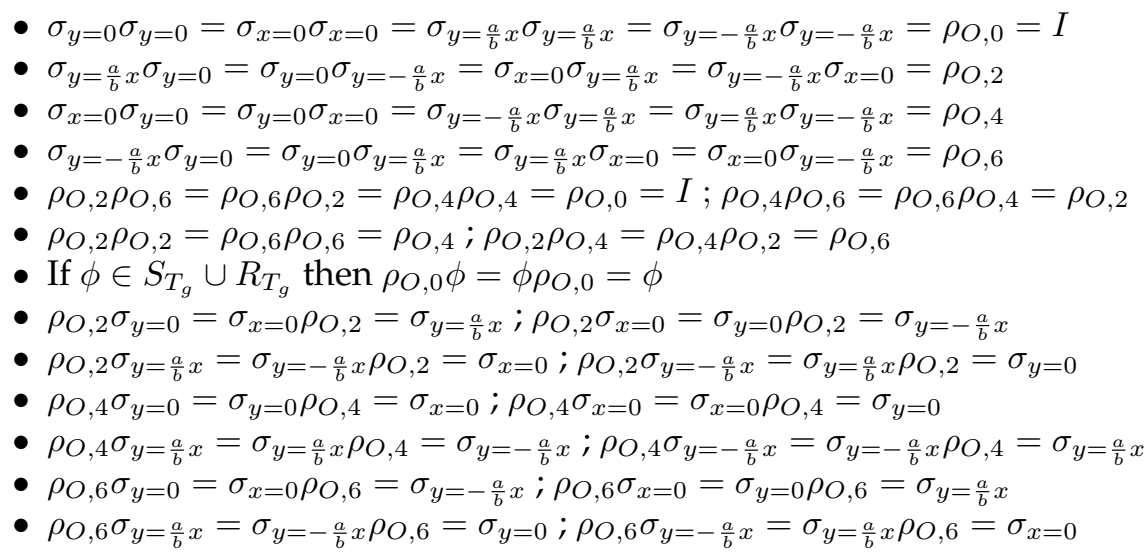

\section{The Generalized Taxicab Isometry Group}

Now, let us consider the image of the unit gt-circle under an isometry fixes the origin: Given an isometry $\phi$ : $\mathbb{R}_{T_{g}}^{2} \rightarrow \mathbb{R}_{T_{g}}^{2}$ such that $\phi(O)=O$. Assume that $\phi\left(A_{1}\right) \in\left(A_{1}, A_{2}\right)$, then $\phi\left(A_{2}\right) \in\left[A_{3}, A_{4}\right]$ since $d_{T_{g}}\left(\phi\left(A_{1}\right), \phi\left(A_{2}\right)\right)=2$. Then $\phi\left(A_{3}\right) \in\left[A_{1}, A_{2}\right]$, and we have $d_{T_{g}}\left(\phi\left(A_{1}\right), \phi\left(A_{3}\right)\right)<2$, which is a contradiction since $d_{T_{g}}\left(A_{1}, A_{3}\right)=2$. Thus, $\phi\left(A_{1}\right) \notin\left(A_{1}, A_{2}\right)$. Using the same way, one gets the fact that $\phi\left(A_{i}\right) \in\left\{A_{1}, A_{2}, A_{3}, A_{4}\right\}$. In addition, if $X \in\left(A_{i} A_{i+1}\right)$ for $i \in\{1,2,3,4\}$ (assume that $\left.A_{5}=A_{1}\right)$, then $d_{T_{g}}(O, \phi(X))=1$ and

$$
\frac{d_{E}\left(A_{i}, X\right)}{d_{E}\left(X, A_{i+1}\right)}=\frac{d_{T_{g}}\left(A_{i}, X\right)}{d_{T_{g}}\left(X, A_{i+1}\right)}=\frac{d_{T_{g}}\left(\phi\left(A_{i}\right), \phi(X)\right)}{d_{T_{g}}\left(\phi(X), \phi\left(A_{i+1}\right)\right)}=\frac{d_{E}\left(\phi\left(A_{i}\right), \phi(X)\right)}{d_{E}\left(\phi(X), \phi\left(A_{i+1}\right)\right)}
$$

by Corollary 1.1 and Corollary 1.2. Thus, $\phi\left(\left[A_{i} A_{i+1}\right]\right)=\left[\phi\left(A_{1}\right) \phi\left(A_{i+1}\right)\right]$ for each $i \in\{1,2,3,4\}$, and so points $O, \phi\left(A_{1}\right)$ and $\phi\left(A_{3}\right)$ (so points $O, \phi\left(A_{2}\right)$ and $\phi\left(A_{4}\right)$ ) are collinear. Therefore, there are 8 possible cases for $\phi\left(A_{1}, A_{2}, A_{3}, A_{4}\right)$ : $\left(A_{1}, A_{2}, A_{3}, A_{4}\right)$, $\left(A_{1}, A_{4}, A_{3}, A_{2}\right)$, $\left(A_{3}, A_{2}, A_{1}, A_{4}\right)$, $\left(A_{2}, A_{1}, A_{4}, A_{3}\right)$, $\left(A_{4}, A_{3}, A_{2}, A_{1}\right),\left(A_{4}, A_{1}, A_{2}, A_{3}\right),\left(A_{3}, A_{4}, A_{1}, A_{2}\right),\left(A_{2}, A_{3}, A_{4}, A_{1}\right)$. It is clear that these conclusions are valid also for a circle $\mathcal{C}$ with center $O$, radius $r$, and vertices $C_{1}, C_{2}, C_{3}, C_{4}$ (labels are as in Figure 1). Moreover, points $\phi\left(A_{i}\right)$ and $\phi\left(C_{i}\right)$ are the same order of above eight possible cases, since $d_{T_{g}}\left(A_{i}, C_{i}\right)=d_{T_{g}}\left(\phi\left(A_{i}\right), \phi\left(C_{i}\right)\right)=|r-1|$. Hence, we can state following proposition using our observations above:

Proposition 5.1. Let $\phi: \mathbb{R}_{T_{g}}^{2} \rightarrow \mathbb{R}_{T_{g}}^{2}$ be an isometry such that $\phi(O)=O$. Then $\phi \in S_{T_{g}}$ or $\phi \in R_{T_{g}}$.

Proof. In the observation above, if $\phi\left(A_{1}, A_{2}, A_{3}, A_{4}\right)=\left(A_{1}, A_{2}, A_{3}, A_{4}\right)$, then $\phi$ is identity. If $\phi\left(A_{1}, A_{2}, A_{3}, A_{4}\right)=$ $\left(A_{1}, A_{4}, A_{3}, A_{2}\right)$, then $\phi$ is the gt-reflection in line $y=0$. If $\phi\left(A_{1}, A_{2}, A_{3}, A_{4}\right)=\left(A_{3}, A_{2}, A_{1}, A_{4}\right)$, then $\phi$ is the gt-reflection in line $x=0$. If $\phi\left(A_{1}, A_{2}, A_{3}, A_{4}\right)=\left(A_{2}, A_{1}, A_{4}, A_{3}\right)$, then $\phi$ is the gt-reflection in line $y=$ $\frac{a}{b} x$. If $\phi\left(A_{1}, A_{2}, A_{3}, A_{4}\right)=\left(A_{4}, A_{3}, A_{2}, A_{1}\right)$, then $\phi$ is the gt-reflection in line $y=-\frac{a}{b} x$. If $\phi\left(A_{1}, A_{2}, A_{3}, A_{4}\right)=$ $\left(A_{2}, A_{3}, A_{4}, A_{1}\right)$, then $\phi$ is the gt-rotation about $O$ through 2 gt-radians. If $\phi\left(A_{1}, A_{2}, A_{3}, A_{4}\right)=\left(A_{3}, A_{4}, A_{1}, A_{2}\right)$, then $\phi$ is the gt-rotation about $O$ through 4 gt-radians. If $\phi\left(A_{1}, A_{2}, A_{3}, A_{4}\right)=\left(A_{4}, A_{1}, A_{2}, A_{3}\right)$, then $\phi$ is the gtrotation about $O$ through 6 gt-radians. Therefore, $\phi \in S_{T_{g}}$ or $\phi \in R_{T_{g}}$.

So, we have the gt-orthogonal group $O_{T_{g}}(2)$ of the real plane with $d_{T_{g}}$ metric, consisting of four gt-reflections and four gt-rotations:

$$
O_{T_{g}}(2)=S_{T_{g}} \cup R_{T_{g}}
$$

which is also the gt-symmetry group of the unit gt-circle. There is one more isometry type having no fixed points: translations. They are the same as Euclidean geometry, since there is no distance or angle notions in the definition:

Definition 5.1. A translation is a mapping $\tau_{v}: \mathbb{R}_{T_{g}}^{2} \rightarrow \mathbb{R}_{T_{g}}^{2}$ such that $\tau_{v}(x, y)=\left(x+v_{1}, y+v_{2}\right)$, where $v=\left(v_{1}, v_{2}\right)$ is a translation vector.

Let $P_{1}=\left(x_{1}, y_{1}\right)$ and $P_{2}=\left(x_{2}, y_{2}\right)$ two points and $v=\left(v_{1}, v_{2}\right)$ is a translation vector, then $P_{i}=\left(x_{i}+v_{1}, y_{i}+v_{2}\right)$ for $i=1,2$ and one gets $d_{T_{g}}\left(\tau_{v}\left(P_{1}\right), \tau_{v}\left(P_{2}\right)\right)=d_{T_{g}}\left(P_{1}, P_{2}\right)$ by using definition of the generalized taxicab distance. So, we can state the following proposition: 
Proposition 5.2. Every translation is an isometry in $\mathbb{R}_{T_{g}}^{2}$.

One can see that we have all isometries of the generalized taxicab plane, by the following theorem:

Theorem 5.1. Let $f: \mathbb{R}_{T_{g}}^{2} \rightarrow \mathbb{R}_{T_{g}}^{2}$ be an isometry. Then there is a unique $\tau_{v} \in T(2)$ and $\phi \in O_{T_{g}}(2)$ such that $f=\tau_{v} \circ \phi$. Proof. Let $f(O)=V$ where $V=\left(v_{1}, v_{2}\right)$. Define $\phi=\tau_{-v} \circ f$. Then, $\phi$ is an isometry and $\phi(O)=O$. Thus, $\phi \in O_{T_{g}}(2)$ by Proposition 5.1, and $f=\tau_{v} \circ \phi$. The proof of uniqueness is trivial.

Finally, we have the generalized taxicab group $T_{g}(2)$ which is semidirect product of the translation group $T(2)$ and the gt-symmetry group of the unit gt-circle $O_{T_{g}}(2)$ (one can use $3 \times 3$ matrix form of the generalized taxicab isometries to show this conclusion as in [12]). The generalized taxicab group $T_{g}(2)$ consists all isometries of the real plane with the generalized taxicab metric, which are all translations, all four isometric gt-reflections, all four isometric gt-rotations and their compositions.

Clearly, if we take $a=b=1$ then all definitions and conclusions given can be adapted for the taxicab geometry.

\section{References}

[1] Akça, Z. and Kaya, R., On the taxicab trigonometry. Jour. of Inst. of Math. Comp. Sci. (Math. Ser.) 10 (1997), no.3, 151-159.

[2] Çolakoğlu, H.B. and Kaya, R., A generalization of some well-known distances and related isometries. Math. Commun. 16 (2011), 21-35.

[3] Ekmekçi, E., Bayar, A. and Altıntaş, A.K., On the group of isometries of the generalized taxicab plane. International Journal of Contemporary Mathematical Sciences 10 (2015), no.4, 159-166.

[4] Ekmekçi, S., Akça, Z. and Altıntaş, A.K., On trigonometric functions and norm in the generalized taxicab metric. Mathematical Sciences And Applications E-Notes 3 (2015), no.2, 27-33.

[5] Gelişgen, Ö. and Kaya, R., The taxicab space group. Acta Math. Hung. 122 (2009), no.1-2, 187-200.

[6] Kaya, R., Akça, Z., Günaltılı, İ. and Ö zcan, M., General equation for taxicab conics and their classification. Mitt. Math. Ges. Hamburg 19 (2000), 135-148.

[7] Kocayusufoglu, İ. and Özdamar, E., Isometries of taxicab geometry. Commum. Fac. Sci. Univ. Ank. Series A1 47 (1998), $73-83$.

[8] Krause, E.F., Taxicab Geometry. Dover, New York, 1986.

[9] Martin, G.E., Transformation Geometry. Springer-Verlag, New York Inc., 1997.

[10] Menger, K., You Will Like Geometry. Guidebook of Illinois Institute of Technology Geometry Exhibit, Museum of Science and Industry, Chicago, Illinois, 1952.

[11] Richard, S. M. and George, D.P., Geometry, A Metric Approach With Models. Springer-Verlag, New York, 1981.

[12] Schattschneider, D.J., The taxicab group. American Mathematical Monthly 91 (1984), no.7, 423-428.

[13] Thompson, K.P. and Dray T., Taxicab Angles and Trigonometry. The Pi Mu Epsilon Journal 11 (2000), no.2, 87-96.

[14] Thompson, K.P., The nature of length, area, and volume in taxicab geometry. International Electronic Journal of Geometry 4 (2011), no.2, 193-207.

[15] Wallen, L.J., Kepler, the taxicab metric, and beyond: An isoperimetric primer. The College Mathematics Journal 26 (1995), no.3, 178-190.

\section{Affiliations}

HARUN BARIŞ ÇOLAKOĞLU

AdDress: Akdeniz University, Vocational Technical School, Computer Technologies, 07070, Antalya, TÜRKIYE.

E-MAIL: hbcolakoglu@akdeniz.edu.tr

ORCID ID : orcid.org/0000-0001-5559-9768 